\title{
Familias transnacionales y política social en las comunidades de origen: una visión cualitativa de la relación entre migración y desarrollo'
}

\author{
MARIANA GABARROT*
}

\begin{abstract}
RESUMEN: Uno de los principales retos para entender la relación entre migración y desarrollo es abordar las realidades específicas de los migrantes y vincularlas con mecanismos más amplios del capitalismo a escala global. Este artículo busca contribuir a esos esfuerzos al proponer como punto de partida el análisis de la experiencia de las familias transnacionales en el marco de la política social, tal y como se concibe dentro del esquema neoliberal, e implementada en el caso mexicano. Se utiliza evidencia de trabajo de campo etnográfico en un municipio rural del estado de Nuevo León, junto con entrevistas detalladas con miembros de familias migrantes y no migrantes para argumentar que comparten elementos comunes, los cuales resaltan las carencias estructurales en materia de trabajo, educación y salud. Lo anterior plantea cuestionamientos importantes hacia la concepción predominante de la política migratoria y la política social en el modelo actual de desarrollo.
\end{abstract}

PALABRAS CLAVE: comunidad de origen, familias transnacionales, política social, capacidades básicas, métodos cualitativos.

\begin{abstract}
One of the key challenges in understanding the relationship between migration and development is in addressing the specific realities of migrants and connect them with the broader processes of capitalism at the global level. This article seeks to contribute to those efforts, proposing as a point of departure the analysis of experiences of transnational families within social policy, as it is conceived within the neoliberal framework, and implemented in the Mexican context. The study makes use of data drawn from ethnographic fieldwork in a rural municipality in the state of Nuevo León, as well as in-depth interviews with members of migrant and nonmigrant families, to identify common, shared elements, which highlight the structural deficiencies in working conditions, education and health care. This leads to significant questions regarding the predominant conception of migratory policy and social policy found within the current development model.
\end{abstract}

KEY WORDS: community of origin, transnational families, social policy, basic capabilities, qualitative methods.

* Profesora titular en el Instituto Tecnológico y de Estudios Superiores de Monterrey en la División de Humanidades y Ciencias Sociales.

${ }^{1}$ Las reflexiones aquí presentadas son parte del proyecto de Ciencia Básica Conacyt sobre «Migración y estrategias de vida en municipios rurales de Nuevo León: el rol de las comunidades de origen en la articulación de los flujos migratorios». 



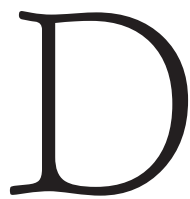

e acuerdo con lo demostrado por los estudios concernientes a migración y desarrollo, ambos procesos ocurren en el marco de una nueva concepción de este último, asociada a la ampliación de libertades individuales. ${ }^{2}$ De tal forma se plantea el fortalecimiento de las capacidades de las personas para "vivir aquella vida que consideran valiosa» (Sen, 1999: 18) y se identifican tres dimensiones clave: vivir una vida larga y sana, tener el conocimiento necesario y un estilo de vida decente. Lo anterior se traduce específicamente en una agenda global de política social, enfocada a la salud, la educación y el ingreso. ${ }^{3}$ Además, se piensa como una cruzada de ayuda a quienes viven en contextos de privación o pobreza.

Dicha concepción neoliberal del desarrollo tiene varias limitantes. Por ejemplo, reduce a una cuestión dicotómica (pobre/no pobre) el complejo debate sobre el bienestar y no contempla libertades como la de tránsito, en cuanto a la migración. Sin embargo, permite adoptar un punto de vista crítico con respecto al modelo predominante, al evidenciar que no ha podido cumplir sus promesas. En términos de capacidades básicas, una proporción importante de la población mundial sigue sin acceso óptimo al ingreso, la educación y la salud. Referente a la mano de obra migrante, lo anterior es cierto a pesar de su movilidad territorial.

La agenda relativa a los derechos humanos ha resaltado en numerosas ocasiones este tema en los países receptores. No ha sucedido lo mismo en los lugares de expulsión, donde los migrantes son parte de los temas invisibles (Delgado, 2013). Ello se debe primordialmente a que los migrantes se han vuelto una fuente de ingresos para sus gobiernos. Muestra de lo anterior, en el caso mexicano, es la preeminencia de los programas 3X1, los cuales privilegian la inversión de migradólares en proyectos productivos, por sobre otros de educación y salud (Fernández, García y Vila, 2006).

\footnotetext{
${ }^{2}$ Mahbub ul Haq, fundador del Reporte de Desarrollo Humano de Naciones Unidas, en http://hdr. undp.org/en/humandev

${ }^{3}$ Estas tres dimensiones comprenden el punto de partida porque han sido incluidas en todas las operaciones de la idea de capacidades. Sin embargo, hay intensos debates en economía y otras ciencias sociales, véase por ejemplo Boltvinik (2003), De Haas (2009), Desai (2003), Pritchett (1997), Sen (1999, 2003a) y Townsend (2003).
} 
A la par de dichas políticas, la mayor parte de los análisis en torno a los impactos de la migración en las comunidades de origen privilegian también sus efectos económicos (Massey, 1988; Nyberg-Sorensen, Van Hear y EngbergPedersen, 2002a, 2002b; Portes, 2009). ${ }^{4}$ Al momento, se ha reconocido la naturaleza celebratoria de esos estudios, al plantear que las remesas en general pueden ser una solución a los dilemas del bienestar, así como la importancia de ir más allá con el objeto de diversificar nuestros enfoques analíticos.

En ese sentido, este trabajo propone un objetivo general: el análisis cualitativo del vínculo entre la migración y el desarrollo (considerado como la ampliación de capacidades básicas), contrastando los impactos de la actual política social con los de la migración a Estados Unidos en las comunidades de origen en México. Para ello, se comparará la lógica de los programas de transferencias condicionadas de dinero con la de las remesas, las estrategias de familias migrantes y no migrantes, beneficiarias de Oportunidades, para asegurar el ingreso, la educación y la salud.

La primera sección del texto desarrolla la metodología utilizada y sus aportes generales, a la vez que resalta la relevancia de la familia y la comunidad de origen dentro de un nivel de análisis para entender la migración y el desarrollo. La segunda presenta una analogía entre el programa Oportunidades y las remesas. La tercera muestra evidencia empírica de esta similitud lógica, basada en entrevistas a profundidad y trabajo etnográfico. Finalmente, se expone una reflexión de las implicaciones del enfoque para desarrollar un punto de vista crítico sobre temas más amplios de la relación entre la migración y el desarrollo.

${ }_{4}^{4}$ Para los programas de educación y salud véase http://www.promocion.salud.gob.mx/dgps/interior1/ programas/vete_sano.html y http://www.sep.gob.mx/es/sep1/sep1_Programa_Binacional_de_ Educacion_Migrante\#.VOdSdbCG_Xo 


\section{Los métodos cualitativos y la familia En la comunidad de origen como punto de partida}

De manera habitual la literatura económica aborda el análisis del hogar como una unidad de corresidencia de personas emparentadas, las cuales comparten un presupuesto común. Sin embargo, los límites de la unidad familiar se definen por los lazos establecidos entre sus miembros, rara vez son los límites de la casa (Esteinou, 2008; Hareven, 2000; Leeder, 2004). Por tal motivo, las relaciones familiares se pueden entender como parte de la vida cotidiana, pues son construidas y reconstruidas a través de prácticas sociales (Bourdieu, 1977; Giddens, 1986). La definición del objeto de estudio se vuelve entonces una pregunta empírica e inductiva. El problema de esclarecer estas microrrutinas de la interacción es un reto, en especial cuando las familias incluyen miembros que viven en territorios distantes (Boehm, 2012; Dreby, 2010; Hondagenu-Sotelo, 1992; Zentgraf y Stoltz, 2012).

Para lograrlo se propone abordar esas relaciones mediante una noción amplia de estrategias de vida, la cual comprende el manejo de «ingresos, tanto en efectivo y otros activos económicos, así como las instituciones (parentesco, familia, barrio, ciudad), género, las relaciones y los derechos de propiedad que se requieren para mantener un determinado nivel de vida [...] también incluyen activos derivados de lo público y de los servicios sociales proporcionados por el Estado, tales como educación, salud, caminos, agua, etcétera» (Ellis, 1998: 4). De tal forma que resulta útil distinguir la familia nuclear (padres e hijos) de la extensa (otros parientes), pero siempre recordando que sus límites se establecen de modo dinámico, conforme las estrategias de vida cambian y las relaciones entre sus miembros se rearticulan.

Así, en el presente trabajo, la familia transnacional no se limita a aquella cuya residencia se halla dividida por la frontera (Ojeda, 2005), también engloba las que cuentan con prácticas transnacionales (Moctezuma, 2011), que pueden implicar, por ejemplo, el uso de remesas o bienes traídos de Estados Unidos o la movilización de sus integrantes (como una hija que acude desde el norte para cuidar a su padre enfermo). Bajo dicha premisa se llevaron a cabo entrevistas en profundidad con 11 familias que han vivido un proceso migratorio 
- es decir, en las que por lo menos uno de sus integrantes ha vivido en Estados Unidos (Moctezuma, 2011) - y las que ostentan una visión transnacional de su reproducción social (planes de regresar, familia extensa o amigos en el norte que forman parte del diseño de las estrategias de supervivencia).

Se incluyeron además ocho familias usuarias de Oportunidades con un perfil similar: cuatro de jefatura femenina e hijos en primaria y secundaria, cuatro familias nucleares con hijos en el mismo nivel de estudios. Todas fueron visitadas dos veces con intervalos de un año, lo que permitió entender longitudinalmente sus experiencias. Es pertinente aclarar que las familias migrantes encontradas en el municipio Doctor Arroyo no son usuarias del programa Oportunidades y viceversa, las familias de Oportunidades no poseen estrategias de vida transnacionales. ${ }^{5}$

Para el análisis interpretativo, se seleccionaron fragmentos de tres entrevistas en familias distintas (dos migrantes y una no migrante) que muestran percepciones coincidentes sobre su realidad inmediata. En ese sentido, se ilustran las generalidades halladas en el material empírico, sin ignorar la heterogeneidad de las experiencias; ello con el fin de resaltar el sentido común, construido alrededor de las capacidades básicas. ${ }^{6}$ Cabe aclarar que el filtro impuesto por la investigación no sólo es producto de la experiencia etnográfica del proyecto, sino de un conocimiento compartido entre investigadores y migrantes a lo largo de los años (Schutz, 1954). P Por tanto, cada sección expone un análisis de los discursos de los entrevistados, los cuales se contextualizan y triangulan con evidencia proveniente de literatura previa y con el material etnográfico.

En cuanto al análisis a nivel local, en términos del porcentaje de población que vive en pobreza de capacidades, la mitad de los municipios del país se

${ }^{5}$ Lo anterior puede indicar que en términos de ingreso, las primeras estén fuera de la línea de pobreza determinada por el cuestionario del programa. Sin embargo, tal como se resaltará en la discusión, no implica que se perciban a sí mismos como distintos.

${ }^{6}$ Con el propósito de construir el argumento de este artículo, se pretendió utilizar material de familias que pertenecieran a la cabecera municipal porque es ahí donde las familias migrantes y no migrantes comparten escuelas y clínicas. No obstante, en toda la muestra se encontraron experiencias similares en el resto de las localidades, con el adicional reto de los traslados hacia la cabecera para ciertas actividades.

${ }^{7}$ Para quien escribe, la experiencia data desde los primeros trabajos de campo realizados en 1994. 
encuentra en un rango de 20 a 40 por ciento y también tienen un grado bajo o medio de intensidad migratoria a Estados Unidos (Consejo Nacional de Evaluación de la Política de Desarrollo Social, 2008; Consejo Nacional de Población, 2010). ${ }^{8}$ En términos muy generales, el perfil de Doctor Arroyo invita a la comparación con cualquiera de ellos, porque 35 por ciento de su población vive en pobreza de capacidades y ostenta un índice medio de intensidad migratoria. Si bien no existe información cuantitativa, el trabajo de campo etnográfico y la literatura correspondiente sugieren que la migración data principalmente desde la época Bracero. En consecuencia, puede compararse con otros municipios donde la tradición migratoria inició en un momento similar (Massey, 1994). Así, se busca construir un argumento basado en las posibilidades de generalización lógica, partiendo de lo local. Es factible que los fenómenos hallados en este contexto sean semejantes a otros que comparten las mismas circunstancias (Bourdieu, Chamboredon y Passeron, 2008).

Por supuesto se reconocen límites, de los cuales se destacan dos: la tradición migratoria de este municipio tiene como destino histórico el valle de Texas; la cercanía territorial facilita la circularidad, tanto documentada como no documentada, principalmente porque puede hacerse vía terrestre (en jornadas que van de 8 a 10 horas), con un costo relativamente bajo. Lo anterior permite la visita frecuente de los migrantes a su lugar de origen y de familiares hacia el destino, así como el envío de regalos. De hecho, existe un servicio de transporte en camionetas desde la cabecera municipal hasta San Antonio y Houston, un fenómeno común en el noreste (Sandoval, 2012).

Asimismo, a pesar de ser un municipio con alta cantidad de localidades rurales, éstas tienen características distintas a las que se sitúan en el centro y sur del país (Appendini y Torres-Mazuera, 2008; Clarke, 2000). Si bien predomina la agricultura de temporal y el cultivo del maíz, hay un grado importante de tecnificación, principalmente el uso del tractor y los pozos para riego. Además, la noción de comunidad no se construye tal cual la encontramos en otras regiones, como Jalisco y Oaxaca (Hirai, 2009; Kearney, 1996). Esto remite a un

${ }^{8}$ Aunque las definiciones de pobreza del Coneval han evolucionado hacia la idea de carencias sociales, los datos a nivel municipal todavía se presentan en esa forma. 
tema complejo, pero cabe mencionar que Doctor Arroyo, al igual que otros municipios en el norte del país, tiene una notable dispersión poblacional, ya que posee 35 mil 445 habitantes y una densidad de 7 por kilómetro cuadrado (Secretaría de Desarrollo Social, 2010). Es probable que ese fenómeno haya sido relevante para articular las costumbres locales alrededor de fiestas relacionadas con la familia, tales como bodas y quince años, o con reuniones relativas a costumbres regionales organizadas en distintas localidades, como jaripeos y cabalgatas (Zúñiga y Reyes, 1988, 2006).

Si bien se alude a la comunidad de origen, es indispensable distinguirla como una referencia hacia la localidad, dado que la identidad no se articula necesariamente de esa forma. A pesar de ello, tal como se apreciará durante el texto, las experiencias familiares parecen hacer eco con otras en territorios distintos (Appendini y Torres-Mazuera, 2008). Dicho municipio también muestra signos de procesos recientes de transformación en el campo mexicano hacia una economía no rural (De Grammont, 2004; Delgado Campos, 1999; Kay, 2007), y la política social tiene características comunes a otras localidades a lo largo del país (Cordera y Ziccardi, 2000).

El trabajo de campo aquí presentado incluye observación y recorridos en un municipio rural del estado de Nuevo León en el mes de junio de 2012, 2013 y 2014. Asimismo, se realizaron entrevistas con informantes clave como campesinos, empleadores y empleados en el comercio, la construcción y la maquila, maestros, doctores, directores de escuela y hospitales, además del presidente municipal. Se asistió a cinco talleres para beneficiarias del programa Oportunidades y se entrevistaron promotoras de salud, vocales y administradores. Lo anterior sirvió para reconstruir las alternativas de ingreso así como la operación cotidiana de la política social, donde se incluye el estado de las escuelas y las clínicas. 


\section{Política social en las comunidades de origen. Transferencias condicionadas y remesas}

Como la mayoría de los países del mundo, México ha sostenido un proceso de crecientes recortes al antiguo Estado de Bienestar (Babb, 2005; Huber, 2003). Los discursos sobre derechos de la ciudadanía social fueron cambiados por los de alivio a la pobreza y por un giro desde las políticas universales a las focalizadas (Gabarrot, 2013; Ordóñez, 2002). Actualmente, se privilegian los programas concentrados en la Secretaría de Desarrollo Social (Sedesol). La mayor parte consiste en transferencias condicionadas de dinero, de las cuales la insignia ha sido Oportunidades; 9 programa enfocado en la reducción de la pobreza alimentaria y de capacidades a través de las madres de familia. Por ejemplo, un hogar puede recibir becas para los niños desde tercer año hasta preparatoria; éstas se encuentran en un rango de 140 a 895 pesos bimestrales, la cantidad aumenta con el grado escolar. A cambio, las beneficiarias deben asegurar la asistencia de los niños a la escuela y la clínica. Oportunidades tiene cobertura territorial amplia, ya que abarca prácticamente todo el México rural y algunos sectores urbanos significativos. Por lo general las evaluaciones han resultado positivas para las familias, pues han mejorado la nutrición y la permanencia en la escuela (Campos, 2010); sin embargo, hay aspectos pendientes: se genera una dependencia del dinero gubernamental, dado que la mayoría de las familias permanece en el programa sin superar las condiciones de pobreza (Cordera, 2007; Martínez y Ziccardi, 2000; Palacios, 2007; Rodríguez, 2007). En ese sentido, se ha resaltado la falta de empleo para los graduados de Oportunidades debido a las condiciones del mercado laboral en las regiones donde opera. Aún hay cuestionamientos específicos sobre la influencia que pueda tener el programa en la permanencia de los estudiantes hacia niveles superiores. También se ha señalado cómo se promueve el uso de escuelas y clínicas sin contemplar su calidad (Campos, 2010; Levy, 2010).

\footnotetext{
${ }_{9}^{9}$ Posterior a la preparación de este artículo el programa se amplió y cambió de nombre a Prospera, no obstante se sostiene el nombre antiguo dado que los principales lineamientos siguen igual. Otros programas dentro de Sedesol incluyen apoyos alimentarios para niños menores de cinco años, subsidios para mejoramiento de la vivienda y microcréditos.
} 
Desde 1992 Durand y Massey mostraron que, además de ser invertida en negocios, una parte importante de las remesas se usa en vivienda, educación y salud. ${ }^{10}$ Resaltaron cómo éstas, ya sea en forma de envíos o de ahorros traídos a México, constituyen una manera de hacer llegar dinero directamente a quienes lo necesitan. Lo anterior ha sido reiterado por estudios subsecuentes (Conway y Cohen, 1998; Durand, Parrado y Massey, 1996; Kanaiapuni y Donnato, 1999). Así, tanto el dinero de Oportunidades como las remesas o migradólares son transferencias focalizadas de efectivo.

Las discusiones sobre los efectos negativos de las remesas en el desarrollo resuenan con las críticas al programa Oportunidades. Desde hace mucho tiempo ha preocupado el hecho de que las comunidades pueden volverse dependientes de las mismas, lo que ocasionaría un síndrome migrante (D. Alarcón, 2003; R. Alarcón, 1992; Reichert, 1981, 1982). De igual modo, se han destacado las limitantes estructurales en los estudios migratorios. Particularmente ha sido señalada la falta de un ambiente propicio para que las remesas generen inversión productiva y empleo (Delgado Wise, García y Márquez, 2006; Taylor, 1999).

Más allá de la similitud en la lógica de operación y las consecuencias, las comunidades de origen sin lugar a duda son territorio de Oportunidades (Prospera, 2014). ${ }^{11}$ La siguiente sección evidencia trabajo de campo etnográfico que muestra otros lugares comunes; ilustra las estrategias de las familias migrantes y las de usuarias de Oportunidades en términos de su percepción acerca de las necesidades de ingreso, educación y salud.

${ }^{10} \mathrm{Si}$ bien la vivienda no se encuentra entre las discusiones de las capacidades básicas, queda claro que es el entorno en el cual éstas se desarrollan y por lo tanto tienen influencia en la calidad de vida. Sin embargo, para efectos de la discusión con la literatura, se ha decidido seguir la consistencia de los debates en el tema, enfocándonos exclusivamente a la educación y la salud. También se muestra cómo esta coincidencia se relaciona con el contexto compartido a nivel municipal.

${ }^{11}$ De hecho, se ha argumentado que el programa puede servir como ancla de potenciales migrantes, al pedir a las familias estar presentes para los chequeos y pláticas de salud (Stecklov et al., 2005).

$154 \frac{\text { SEGUNDO SEMESTRE } 2016}{\text { MIGRACIÓN Y DESARROLLO NÚM. } 27}$ 


\section{Más allá del dinero: el discurso de la escasez}

En su mayoría, los estudios microeconómicos sobre las remesas han demostrado que las mismas aumentan el ingreso de los hogares, diversificando su gasto (Stark y Lucas, 1988; Taylor, 1999). Por su parte, la literatura sobre política social busca principalmente calcular los ingresos necesarios para que la familia, como unidad económica, pueda salir de la pobreza (Boltvinik, 2003; Consejo Nacional de Evaluación de la Política de Desarrollo Social, 2008; Cornia y Stewart, 2003; Desai, 2003). Así, tanto las remesas como las transferencias condicionadas son vistas como un insumo, el cual tiene impactos positivos en la calidad de vida presente o futura de los hogares. Sin embargo, la diferencia en términos de la percepción de insuficiencia o de dificultad económica no es tan obvia. Consideremos la experiencia de tres familias ilustradas por los siguientes fragmentos de entrevista:

Dos mil pesos por quincena żqué haces? En las vacaciones que vienen en julio y agosto... no hay entrada de dinero; mi esposo tenía herramienta que nos trajimos de allá, la ha estado vendiendo pero como es herramienta cara, pues le dan de doscientos pesos, de quinientos, así, cualquier cosa. Dices bueno, doscientos ya paga el agua, que trescientos que paga el teléfono, que paga la luz, que paga esto, pero para comer, nada más lo mío (Ana, familia nuclear migrante de retorno reciente con familia extensa en Estados Unidos).

Yo traigo [ollas] las que valen 17 y las vendo aquí en Doctor Arroyo, las doy en 9 mil 500 y en pagos. Entonces pues yo con que venda una la hago. Mi señora está aquí trabajando por la presidencia, le dan 900 pesos a la quincena; a mí me puede ver que estoy muy fresco y lo que sea, no ando haciendo nada, debería andar trabajando allá [en Estados Unidos] pero yo le busco la manera, ya acomodo una batería y me dura para varios meses. (Jesús, migrante circular de familia nuclear en México y familia extensa en Estados Unidos).

No, yo no, yo me lo quedo [el dinero de Oportunidades], yo lo guardo y eso no es para alimentación, no es para eso. Sí agarro un poquito, pero la mayor parte es para ellas: para sus uniformes, para su colegiatura, para lo que les piden, para 
zapatos, para que vayan bien presentables. Para eso es para lo que lo utilizo (Lupita, beneficiaria del programa Oportunidades).

Tal como se argumentará a lo largo de esta sección los perfiles son diferentes, pero todos apuntan a dinámicas sociales más generales. Podemos entonces triangularlos con distintos cuerpos de literatura. El primero lo constituyen los estudios de género: ambas mujeres son más específicas en su descripción del gasto doméstico, porque de alguna manera son ellas las directamente responsables de ejercerlo, al ser las encargadas de lo privado (Hondagenu-Sotelo, 1992; Moller, 1998; Pessar, 1999, 2001; Pessar y Mahler, 2003). El segundo elemento es la desemejanza entre familias migrantes y no migrantes, las primeras tienen un recurso específico proveniente de Estados Unidos: la herramienta del esposo de Ana y las ollas que trae Jesús desde Houston para vender (Durand, $\mathrm{Pa}$ rrado y Massey, 1996; Gabarrot, 2009; Portes, 2009; Sørensen y Olwig, 2002). Por último, las tres entrevistas hacen clara referencia a elementos de la estrategia familiar donde se manejan activos para la reproducción social de todos los miembros (Taylor, 1999). La escasez es un aspecto que surge constantemente en la comunidad de origen.

Los patrimonios son estrechos y se gastan con cuidado, los cálculos son minuciosos, con cantidades y rubros específicos, siempre hay necesidad de más. El escenario en el cual esta situación pudiera cambiar es también tema de muchos debates, pero claramente es un asunto de largo plazo, donde la inmediata falta de esos recursos implicaría un desbalance importante para la estructura de ingreso/gasto, más allá de la cantidad concreta que se recibe. En ese sentido, las familias perciben dependencia tanto de las remesas como de los programas sociales.

Es indispensable resaltar que la referencia a la escasez se encuentra a pesar de que las familias migrantes no son beneficiarias del programa. Ana y Jesús, al preguntarles por qué no han sido seleccionados, contestan de manera ilustrativa a todos los demás entrevistados para el proyecto: 
Es lo que te digo, mira aquí en la escuela no sé dónde se dan esas, hay mucha información que yo todavía no sé; ahora, creo que estaba un programa pero te ven aquí, por ejemplo el apellido de nosotros es Banda Ortiz, te ven el apellido y dicen «ah, éste no lo necesita» (Ana). No, pues vienen aquí y miran la casa y dicen "no, éste no necesita», lo que necesitan es ver una casa que esté... no sé, con lámina y así pues sí, yo ni me quejo, digo para que me voy a quejar, pero si nos ayudan bueno (Jesús).

La idea de no recibir un apoyo gubernamental es atribuida a una arbitrariedad, o a una injusticia, más que a una sensación de bienestar o de no necesitarlo. Esta noción de requerir —en sus propias palabras — «ayuda», se refleja también en el discurso de Lupita, aunque en tal caso ella se refiera a por qué sí le dan Oportunidades: «Oportunidades... nos dan ese beneficio por decir, como... ellos andan haciendo un estudio socioeconómico casa por casa, entonces es cuando ahí ellos te detectan que si necesitas ayuda, que si tienes hijos para estudiar».

Estas oraciones muestran la importancia de la percepción - la cual va mucho más allá de los cálculos específicos acerca de su situación económicaderivada de un contexto común, donde las opciones son limitadas. Tal como se mencionó, Doctor Arroyo es un municipio agrícola; es una región históricamente productora de lechuguilla (fibra similar al henequén), la cual se vende en 12 o 13 pesos el kilo. La productividad del campo es en general baja, debido a la falta de agua común en la región (Contreras, 2007). Diagnósticos recientes cuestionan la clasificación de dicha zona como predominantemente agraria (Instituto Nacional de Geografía e Informática, 2011), dado que esa actividad pareciera pasar a un segundo plano en comparación con el sector servicios (Consejo de Desarrollo Social de Nuevo León, 2009). Además, durante el trabajo de campo no se halló a nadie que se dedicara al cien por ciento a aquella actividad, por lo que funge como complemento de los ingresos.

Otras fuentes de empleos locales incluyen: primero, una maquiladora instalada en la cabecera municipal, donde se ensamblan partes de arneses automotrices para exportación; segundo, jornales o pequeñas propiedades en viveros para la producción y venta de tomate, instalados con subsidios del gobierno estatal; tercero, empleos en el sector comercial o de servicios tales como trabajo 
doméstico, peluquerías y talleres mecánicos. Los sueldos tienen un rango de 90 a 160 pesos diarios y la mayoría no ofrece seguridad social.

En el caso específico de los entrevistados, Ana utiliza su conocimiento del inglés aprendido en Estados Unidos para dar clases particulares, mientras su esposo trabaja esporádicamente como jornalero en la construcción. Jesús vende ollas y alterna esta actividad con la migración circular, en tanto su esposa tiene un empleo temporal como secretaria en un proyecto de la Secretaría de Medio Ambiente. Lupita es dueña de una papelería y su esposo labora en la construcción. Las historias revelan un contexto compartido donde las pocas opciones son de trabajo precario, tal como lo demuestran las evidencias relativas a la educación y la salud.

A grandes rasgos los estudios sobre educación y migración han sido enfocados a la integración/adaptación de los niños al sistema escolar de las comunidades de destino (Valdés, 1996). Sin embargo, existe literatura más reciente sobre las implicaciones que tiene la circularidad migrante para los niños que deben estudiar en escuelas de ambos países. Así, se han mostrado problemáticas específicas de aprendizaje, las cuales plantean retos importantes de integración y desempeño escolar (Hamann, Zúñiga y Sánchez, 2008; Zúñiga y Sánchez, 2010).

Por su parte, la salud de los migrantes ha sido principalmente analizada desde el punto de vista del acceso a la atención médica, ya sea en general o con respecto a temas específicos tales como la salud reproductiva o la salud mental (Brown, 2008; Calvo, 2008; Infante, 1990; Ochoa-Marín, Cristancho-Marulanda y González-López, 2011; Ramos, 2010). Algunos estudios se han enfocado en el estado de salud de los migrantes que retornan a México (González-Block y De la Sierra-De la Vega, 2011; Ullmann, Goldman y Massey, 2011) o específicamente en el impacto de la migración en la salud infantil de los niños en la comunidad de origen (Hilderbrandt y McKenzie, 2005; Kanaiapuni y Donnato, 1999).

En la literatura sobre política social y desarrollo es imposible sintetizar los trabajos acerca de estos temas porque constituyen la parte medular de la definición del concepto. Expresado de otra forma, no hay desarrollo sin educación y sin salud. Así, hay trabajos especializados en cada área y aquellos que abor- 
dan en conjunto, desde la discusión de las capacidades básicas (Moser, 1998; Sen, 1999, 2003b; Toye, 2003).

Hay una narrativa clara en todas las familias entrevistadas. Por un lado, hay referencias generales al uso del dinero para sostener la familia, lo cual comprende entre otros elementos, asegurar la educación primaria de los hijos. Por otro, existe una evidente preocupación con respecto a la continuidad de los estudios, que se refleja en un discurso donde los padres se comprometen en ese sentido. Ana, Jesús y Lupita lo manifiestan de la siguiente forma:

Él está en enfermería. Y ahorita anda mal porque es no sé, no sé, le digo, a veces me pongo a platicar con él, le digo żen qué estoy fallando yo, mijo? No sé. Porque nada más es con él. No sé si sea la edad, no sé. Mira, pasó primer año, segundo año y este semestre me lo reprobó. Entonces se tuvo que quedar otra vez, otro año. ¿Qué está pasando contigo? Si no hubieras entendido el español luego luego, me hubieras reprobado el primer año. Pero me estás reprobando lo último (Ana).

Le digo «échele ganas hijo», nomás que sí se me había puesto rebelde. Lo llevé a trabajar a la construcción, «vente a pintar» le dije, como quiera te pago bien, más que a los trabajadores y pues iba tarde y una vez de las que vino, se vino a comer y ya no salió... regresó [a la escuela] (Jesús). Pues ojalá y que ellas quieran seguir adelante, yo las agarro a consejos, porque les digo: «hijas, żqué más les puedo dar? más que un estudio; uno no sabe qué suerte les vaya a tocar, pero ustedes ya van a tener un respaldo con su estudio» (Lupita).

Estas tres familias (como muchas otras en Doctor Arroyo) tienen un hijo o hija en los primeros años de educación técnica. En el caso de Ana, además de un hijo estudiando enfermería en el Conalep, tiene un niño en primaria y una hija en secundaria. Por su parte, el hijo de Jesús (mayor de tres, una hermana en secundaria y otro hermano en primaria) se encuentra en los primeros años de la escuela normal. La hija de Lupita se hallaba en proceso de entrar a la carrera de enfermería durante la entrevista, e ingresó durante nuestra segunda visita (su hermano y hermana menor están en primaria). A lo largo de nuestras conversaciones los padres hablaron sobre el reto que esto representa en términos de la economía familiar, así, se considera el gasto como un sacrificio, el cual vale la pena. 
Es interesante que en el caso de Ana, como migración de retorno reciente, su hijo pareciera presentar los problemas de aprendizaje derivados de la readaptación, ya documentados en la literatura. Sin embargo, a pesar de este diferenciador obvio, la preocupación y la construcción subjetiva del valor de la educación siguen siendo las mismas. Además, todos los niños comparten escuelas primarias y secundarias en la cabecera municipal, lo cual implica que de entrar en una discusión sobre la calidad educativa, el contexto sería el mismo para familias migrantes y no migrantes (Hamann, Zúñiga y Sánchez, 2006).

El Conalep es la opción en ambos casos discutidos, pero también la Universidad Tecnológica de Linares ofrece carreras técnicas en la cabecera. En contraste, de querer continuar a la universidad, las familias tendrían que optar por enviar a sus hijos a una de las dos ciudades más cercanas: San Luis Potosí o Monterrey. Por lo general, quienes deciden y pueden seguir estudiando son migrantes potenciales hacia zonas urbanas; una vez graduados es probable que permanezcan en ellas, debido a las pocas opciones de empleo mencionadas en la sección anterior. Esto refuerza la idea de que el desarrollo genera en primera instancia migración (De Haas, 2005); al mismo tiempo, apunta nuevamente hacia la limitante de la migración para generar desarrollo a nivel local, si no existen las condiciones para ello.

Con respecto al tema de salud, una necesidad de atención médica es responsabilidad de toda la red familiar, incluyendo la red de familia extensa, tal como lo muestra el caso de los entrevistados:

Llegó esa prima de mi esposo ahí, se embarazó ahí llegando y tú le tomabas el pulso y no tenía pulso. La enfermedad... me dice... le digo «te voy a llevar a checar después de los seis meses, para que no te cobren tanto en el hospital, pero si quieres de una vez empezamos, si tú te sientes mal o tienes algún síntoma tú me vas a decir y yo te llevo inmediatamente al hospital». Bueno, dice «no, sí me aguanto, nomás los ascos, los ascos». A los seis meses la llevé y las doctoras empezaron a moverse, dije «żqué le pasa?», dice «es que está como muerta» (Ana)

[...] pues ya... en la tarde como a las 3 les dije «no, żsabes qué?, me siento bien mal, tengo que ir a la clínica», le dije, "voy a quedarme haciendo el fuerte y me va a salir peor». No pues ya ahí... estaban los hermanos que tengo allá en 
Dallas... no en Dallas, es en medio de Dallas y Forthworth, que es Real Pearl y Arlington. Allí tengo unos hermanos. Y ya fue uno de ellos a levantarme... llegamos a la casa y ya me recosté un rato. Al siguiente día me llevaron en la mañana... mi hermana, ya le tocó a mi hermana, ella me llevó a buscarme una clínica, porque va uno a cualquier clínica de allá y es un dineral, hasta por una pastilla es mucho dinero lo que cobran (Jesús).

Estuve obligada a ir [a Monterrey] dos años, dos años seguidos de aquí para atrás, porque mi niña chiquita tuvo un problemita: le salieron muchas manchitas en su cuerpo, como moretones, entonces yo me asusté porque aquí me dijeron «puede ser leucemia, puede ser una enfermedad grave». Me dieron el pase al [Hospital] Universitario de Monterrey, entonces yo estuve yendo allá por dos años cada mes, cada mes, cada mes. Entonces, era cuando ya la veía yo [a mi hermana], nos veíamos más seguido porque iba yo y a los quince días venía ella y así iba yo y así (Lupita).

Los extractos de estas entrevistas refieren situaciones diversas. En el caso de Ana, su cuñada tuvo problemas con el embarazo; Jesús mostraba señales de prostatitis y la hija menor de Lupita tenía anemia severa. Asimismo, en ambas experiencias migratorias, las citas aluden a una situación acontecida en Estados Unidos. La primera enfrenta una barrera de idioma y Ana decide acompañarla porque ella habla inglés (Chávez, 1984). El segundo buscó una clínica de beneficencia para que le saliera más barato el servicio (Portes, Fernandez-Kelly y Light, 2012). Para los tres entrevistados el tema obvio es el rol solidario de los hermanos. En ese sentido, ilustran perfectamente cómo la familia extensa juega un rol importante en las estrategias de vida cuando se trata de enfrentar situaciones extraordinarias. Esto nos remite a la idea de la familia como una construcción dinámica, es decir, puede ser que durante la enfermedad se fortalezcan lazos entre los miembros.

En el caso de Jesús, uno de los motivos para su retorno fue la detección de una hernia, ante lo cual decidió operarse en Doctor Arroyo. Como se observa, la experiencia con los servicios de salud varía conforme a elementos coyunturales: tipo de enfermedad o relación con el personal de la clínica. Por ejemplo, mientras Jesús considera su operación como un éxito, gracias a que le tocó un 
muy buen doctor y Lupita comparte esa opinión positiva, Ana prefiere ir a un médico particular. Sin embargo, los servicios de salud de Doctor Arroyo comparten las características comunes de zonas rurales: escasez de medicamentos y hospitales de primer nivel; motivo por el que hay que trasladarse fuera del municipio en caso de requerir otro tipo de atención (Frenk y Gómez, 2008; Linares-Pérez y López-Arellano, 2012). Lo anterior engloba otro tema relativo a la salud y la política social.

Dado que las alternativas de trabajo son limitadas, la población con acceso a seguridad social es mínima (11 por ciento). De todas formas, las cifras advierten una cobertura amplia en el municipio, gracias a la protección básica ofrecida por el Seguro Popular (más de 76 por ciento) (Instituto Nacional de Estadística, Geografía e Informática, 2011). No obstante, esto de ninguna manera asegura el acceso a los hospitales, puesto que la ubicación y las posibilidades de transporte se vuelven tan importantes como el hecho de estar o no cubierto. Vale la pena resaltar que todos los entrevistados tienen el mismo tipo de seguro (popular).

De acuerdo con las particularidades de cada historia, hay elementos compartidos no sólo por el resto de las familias en Doctor Arroyo, sino también por otras en distintos contextos. Lo anterior ha sido señalado al vincular estos casos ilustrativos de una realidad local, a otros documentados en la literatura. Ello arroja luz sobre aspectos importantes de la relación entre migración y desarrollo desde la experiencia subjetiva y en su dimensión de capacidades, lo cual puede sugerir algunas avenidas de análisis a futuro. Asimismo, es posible llevar a cabo una reflexión crítica del modelo neoliberal predominante. La siguiente sección ofrece reflexiones en términos de cómo los resultados muestran una realidad y un sentido común que pueden servir de punto de partida. 


\section{A manera de conclusión: ¿qué nos dice la política social sobre la migración y viceversa?}

Las similitudes en la lógica y los impactos de las transferencias tanto de remesas como de Oportunidades, discutidas en la primera sección, pueden entenderse dentro del marco de la agencia en contextos estructuralmente restringidos. Tal como se muestra en las historias de familia, existe una preocupación que guía las estrategias familiares y la construcción de una visión de futuro, donde la educación es un tema fundamental. Por su parte, la salud refleja una resolución de problemas donde la red nuclear se amplía para involucrar a distintos familiares según las circunstancias. Para entender el impacto que tienen las transferencias de dinero es necesario prestar atención de manera diacrónica e integral a los activos movilizados a lo largo del tiempo, no sólo económicos sino también sociales.

Lo anterior expone la utilidad de la noción de estrategias de vida, al permitir el análisis de la relación que tienen las unidades familiares con el entorno institucional al cual acuden: fuentes de trabajo, escuelas y clínicas. De tal forma se puede entender la especificidad de los migrantes y sus redes en el marco de las problemáticas que comparten con sus vecinos en la comunidad de origen. Entonces, parece posible estudiar los impactos de la migración en el desarrollo, más allá de las remesas; e incluso sin enfocarse necesariamente en ellas.

Se refuerza la idea de que los migrantes son objeto de exclusión y que sus derechos sociales tampoco están cubiertos en la comunidad de origen. La precariedad de las condiciones de vida mostradas en el material empírico se suma a la precariedad de las condiciones laborales ofrecidas para los trabajadores migrantes a nivel global. Esto corrobora la idea de que los flujos son producto de la desigualdad producida y reproducida por el modelo capitalista actual (Canales, 2013; Castles y Delgado Wise, 2007; Delgado Wise y Covarrubias, 2008). Parece imposible pensar en una agenda exclusivamente migratoria, a la vez que se refuerza la noción de retomar una visión amplia de reivindicación de derechos humanos y dignificación del trabajo en general.

Las experiencias analizadas manifiestan la necesidad de una mejora en la política social en México. En cuanto al funcionamiento mismo del programa 
Oportunidades, los fragmentos donde Ana y Jesús indican su extrañeza ante la lógica de la asignación de recursos, hacen eco no sólo a nivel local, sino a nivel nacional (Vizcarra y Guadarrama, 2008). Más allá de las debilidades propias del programa, es evidente que las transferencias se encuentran lejos de resolver las inquietudes percibidas por los beneficiados.

Una explicación plausible, al considerar en términos de la literatura revisada, es que esas inquietudes reflejan problemas de fondo a los cuales se enfrentan las poblaciones marginadas, tanto en México como a nivel global. Es indispensable considerar que representan la experiencia a nivel microsocial, a la vez que reflejan realidades macrosociales, y que ambas son parte de un mismo proceso de estructuración de la realidad (Giddens, 1986; Bourdieu, 1977). Se fortalece la noción de que es un error ver al programa Oportunidades y a las remeses como la panacea del desarrollo, sin analizar las condiciones relacionadas a la generación de empleo, la calidad de los sistemas de educación y de salud. Una política migratoria enfocada a la atracción de remesas y su inversión, así como una política social focalizada, están limitadas severamente, sin un acompañamiento de inversión en materia social, con una participación sustancial de dinero público. Para que eso suceda, el desarrollo tiene que ser discutido de manera más integral y no sólo como un conjunto de libertades individuales.

Finalmente, los paralelos encontrados entre las familias de Doctor Arroyo y otros temas generales en la literatura, entrañan que la dinámica del capitalismo moderno acerca, en términos de exclusión y carencias, a territorios antes distantes, por lo menos en el caso mexicano. Si bien el análisis específico de la ruralidad va más allá de los objetivos de este trabajo, da la impresión de que los procesos aquí señalados son similares a otros fuera de la región norte. El municipio muestra signos de lo que se ha discutido en el marco de la nueva ruralidad. Las actividades agropecuarias han pasado a segundo plano ante la penetración de actividades que generan trabajo precario y lo vinculan con procesos económicos a nivel nacional y global (por ejemplo la penetración de la maquila y los viveros de exportación) (De Grammont, 2001; Delgado Campos, 1999; Kay, 2009). Sin embargo, es esencial recordar que esos fenómenos tienen implicaciones muy concretas para quienes construyen el día con día frente a tales cambios. En ese 
sentido, este trabajo resalta la importancia de identificar elementos desde lo cotidiano y desde la experiencia subjetiva, los cuales pueden ayudar a dar cuenta de la complejidad de las distintas realidades de la migración y el desarrollo, al mismo tiempo que se vinculan con discursos más amplios, tanto en los ámbitos académicos como en las agendas de derechos humanos.

\section{Bibliografía}

Alarcón, D. (2003), «Income Distribution and Poverty Alleviation in Mexico: A Comparative Analysis», en K. J. Middlebrook y E. Zepeda (eds.), Confronting Development: Assesing Mexico's Economic and Social Policy Challenges, México, Secretaría de Desarrollo Social.

AlarCón, R. (1992), «Norteñización: Self-Perpetuating Migration from a Mexican Town», en J. A. Bustamante, C. W. Reynolds y R. A. Hinojosa Ojeda (eds.), U.S.Mexico Relations: Labor Market Interdependence, California, Stanford University Press.

ApPendini, K. y G. Torres-Mazuera (2008), «Perspectivas multidisciplinarias de una realidad fragmentada», en K. Appendini y G. Torres-Mazuera (eds.), żRuralidad sin agricultura?, México, El Colegio de México.

BABB, S. (2005), «The Social Consequences of Structural Adjustment: Recent Evidence and Current Debates», Annual Review of Sociology, vol. 31.

BoeHm, D. A. (2012), Intimate Migrations: Gender, Family, and Illegality Among Transnational Mexicans, Nueva York, New York University Press.

BoltVinik, J. (2003), "Conceptos y métodos para el estudio de la pobreza», Comercio Exterior, vol. 53, núm. 5.

Bourdieu, P. (1977), Outline of a Theory of Practice, Cambridge, Cambridge University Press.

Bourdieu, P., J. C. Chamboredon y J. C. Passeron (2008), El oficio del sociólogo, México, Siglo XXI Editores.

BRown, H. S. (2008), "Do Mexican Immigrants Substitute Health Care in Mexico for Health Insurance in the United States? The Role of Distance», Social Science y Medicine, vol. 67. 
Calvo, J. M. (2008), "The Consequences of Restricted Health Care Access for Immigrants: Lessons from Medicaid and SCHIP, Annals of Health Law, vol. 17, no. 2.

CAMPOS BOlaño, P. (2010), Documento compilatorio de la evaluación externa 2008 del Programa Oportunidades, en http://www.oportunidades.gob.mx/ EVALUACION/es/docs/docs_eval_2010.php (recuperado el 31 de mayo de 2012).

CANALES, A. I. (2013), "La migración en la reproducción de la sociedad global», Migración y Desarrollo, vol.12, segundo semestre.

CAStles, S. y R. Delgado Wise (2007), Migración y desarrollo: perspectivas desde el sur, México, Miguel Ángel Porrúa.

Chávez, L. R. (1984), «Doctors, Curanderos and Brujas: Health care Delivery and Mexican Immigrants in San Diego", Medical Anthropology Quarterly, vol. 15, no. 2.

Clarke, C. G. (2000), Class, Ethnicity, and Community in Southern Mexico: Oaxaca's Peasantries, Oxford, Oxford University Press.

Consejo de Desarrollo Social de Nuevo León (2009), «Diagnóstico de las familias del sur de Nuevo León, Monterrey, Nuevo León, Consejo de Desarrollo Social de Nuevo León. Consejo Nacional de Evaluación de la Política de Desarrollo Social (2008), Informe de Evaluación de la Política de Desarrollo Social en México 2010», en http://medusa.coneval.gob.mx/

Consejo Nacional de Población (2010), «Índice de intensidad migratoria», en http:// www.conapo.gob.mx/work/models/CONAPO/intensidad_migratoria/pdf/II_ Generales.pdf (11 de junio de 2012).

Contreras, C. (2007), Geografía de Nuevo León, Monterrey, México, Fondo Editorial de Nuevo León.

CONWAY, D. y J. Cohen (1998), "Consequences of Remmittances for Mexican Transnational Communities», Economic geography, vol. 74, no. 1.

Cordera CAmpos, R. (2007), «Mercado y equidad: de la crisis del Estado a la política social», en R. Cordera Campos y C. J. Cabrera Adame (eds.), La política social en México: tendencias y perspectivas, México, Universidad Nacional Autónoma de México.

Cordera Campos, R. y A. Ziccardi (eds.) (2000), Las politicas sociales de México al fin del milenio. Descentralización, Diseño y Gestión, México, Miguel Ángel Porrúa y Universidad Nacional Autónoma de México. 
CorniA, G. A. y F. Stewart (2003), «Subsidios alimentarios: dos errores de focalización», Comercio Exterior, vol. 53, núm. 5.

De Grammont, H. (2001), "El campo mexicano a finales del siglo XX», Revista Mexicana de Sociología, vol. 63, núm. 4. (2004), "La nueva ruralidad en América Latina», p. 279.

DE HAAS, H. (2005), «International Migration, Remittances and Development: Myths and Facts», Third World Quarterly, vol. 26, no. 8. (2009), «Mobility and Human Development», Human Development Research Paper, en http://hdr.undp.org/sites/default/files/hdrp_2009_01_rev.pdf

DELGADO CAMPOS, Javier (1999), "La nueva ruralidad en México», Investigaciones Geográficas, núm. 39.

DELGADO Wise, R. (2013), «Diez años de promover una agenda alternativa de investigación y acción», Migración y Desarrollo, vol. 11, núm. 20.

Delgado Wise, R. y H. M. Covarrubias (2008), «Capitalist Restructuring, Development and Labour Migration: The Mexico-US Case», Third World Quarterly, vol. 29, no. 7.

Delgado Wise, R., R. García Zamora y H. Márquez Covarrubias (2006), "México en la órbita de la economía global del trabajo barato: dependencia crítica de las remesas», Theomai, vol. 14, segundo semestre.

DESAI, M. (2003), «Pobreza y capacidades: hacia una medición empíricamente aplicable», Comercio Exterior, vol. 53, núm. 5.

DreBY, J. (2010), Divided by Borders: Mexican Migrants and their Children, Berkeley, University of California Press.

DuRAnD, J., E. A. Parrado y D. S. Massey (1996), «Migradollars and Development: A Reconsideration of the Mexican Case», International Migration Review, vol. 30, no. 2.

ELLIS, F. (1998), «Household Strategies and Rural Livelihood Diversification», The Journal of Development Studies, vol. 35, no. 1.

ESTEINOU, R. (2008), La familia nuclear en México: lecturas de su modernidad siglo XVI al XX, México, Miguel Ángel Porrúa.

FERnÁNDEZ De CASTRO, R., R. García Zamora y A. Vila Freyer (2006), El programa $3 \times 1$ para migrantes: primera politica transnacional en México?, México, Instituto Tecnoló- 
gico Autónomo de México, Universidad Autónoma de Zacatecas y Miguel Ángel Porrúa.

Frenk, J. y O. Gómez Dantés (2008), El sistema de salud en México, México.

GABARROT ARENAS, M. (2009), "Capital social y política social en comunidades migrantes: reflexiones desde dos comunidades en los valles centrales de Oaxaca», en S. Berumen Sandoval y J. Arroyo Alejandre (eds.), Autoempleo, programas sociales y migración a Estados Unidos: propuestas para políticas públicas, Guadalajara, Universidad de Guadalajara.

(2013), «Algunas contradicciones en la política social mexicana. Bienestar y combate a la pobreza», Trace, vol. 62.

Giddens, A. (1986), The Constitution of Society, Cambridge/Oxford, Polity Press.

GonZÁlez-Block, M. A. and L. A. De la Sierra-De la Vega (2011), «Hospital Utilization by Mexican Migrants Returning to Mexico due to Health Needs», BMC Public Health, vol. 11, suplemento 4.

Hamann, E., V. Zúñiga y J. Sánchez García (2006), «Pensando en Cinthia y su hermana. Educational Implications of United States-Mexico Transnationalism for Children», Journal of Latinos and Education, vol. 5, no. 4. (2008), Alumnos transnacionales, escuelas mexicanas frente a la globalización, México, Secretaría de Educación Pública.

Hareven, T. K. (2000), Families, History, and Social Change: Life Course and Cross-Cultural Perspectives, Westview Press.

HilderbrandT, N. and D. J. McKenzie (2005), «The Effects of Migration in Child Health in Mexico", World Bank Policy Research Working Paper.

HirAI, S. (2009), Economía política de la nostalgia: un estudio sobre la transformación del paisaje urbano en la migración transnacional entre México y Estados Unidos, México, Departamento de Antropología de la UAM-I y Juan Pablos Editor.

Hondagenu-Sotelo, P. (1992), «Overcoming Patriarchal Constraints: The Reconstruction of Gender Relations among Mexican Immigrant Women and Men», Gender and Society, vol. 6, no. 3.

Huber, E. (2003), "Opciones de política social para América Latina: modelos neoliberales y socialdemocracia», Comercio Exterior, vol. 53, núm. 6.

INFANTE, C. (1990), "Utilización de servicios de atención prenatal: influencia de la morbilidad percibida y de las redes sociales de ayuda», Salud Pública Mex, vol. 32. 
Instituto Nacional de Estadística, Geografía e Informática (2011), http://www.inegi. org. $m x$ (12 de octubre de 2011).

KanAiApuni, S. and K. Donnato (1999), "Migradollars and Mortality: the Effects of Migration on Infant Survival in Mexico», Demography, vol. 43, no. 3.

KAY, C. (2007), «Algunas reflexiones sobre los estudios rurales en América Latina (spanish)", Some Reflections on Rural Studies in Latin America (english), núm. 29.

(2009), «Estudios rurales en América Latina en el periodo de globalización neoliberal: ว̇una nueva ruralidad?’», Revista Mexicana de Sociología, núm. 4.

KeARneY, M. (1996), Reconceptualizing the Peasantry: Anthropology in Global Perspective, Oxford, Westview.

LEEDER, E. J. (2004), The Family in Global Perspective: a Gendered Journey, Thousand Oaks, California, Sage Publications.

LEVY, S. (2010), Buenas intenciones, malos resultados. Política social, inconformidad y crecimiento económico en México, México, Océano.

LinARES-PÉREZ, N. y O. López-Arellano (2012), "Inequidades en la salud en México», Gaceta Médica de México, vol. 148.

MARTÍneZ ASSAD, C. y A. Ziccardi (2000), «Límites y posibilidades para la descentralización de las políticas sociales», en R. Cordera Campos y A. Ziccardi (eds.), Las políticas sociales de México al fin del milenio. Descentralización, diseño y gestión, México, Miguel Ángel Porrúa y Universidad Nacional Autónoma de México.

Massey, D. B. (1994), Space, Place and Gender, Cambridge, Polity.

MASSEY, D. S. (1988), "Economic Development and International Migration in Comparative Perspective», Population and Developement Review, vol. 14, no. 3.

Moctezuma Longoria, M. (2011), La transnacionalidad de los sujetos. Dimensiones, metodologías y prácticas convergentes de los migrantes mexicanos en Estados Unidos, México, Universidad Autónoma de Zacatecas y Miguel Ángel Porrúa.

Moller OKin, S. (1998), "Gender, the Public and the Private», en A. Phillips (ed.), Feminism and Politics, Oxford, Oxford University Press.

Moser, C. (1998), "The Asset Vulnerability Framework: Reassesing Urban Poverty Reduction Strategies», World Development, vol. 26, no. 1.

NyberG-Sorensen, N., N. Van Hear and P. Engberg-Pedersen (2002a), "The Migration- Developement Nexus Evidence and Policy Options», International Migration, vol. 40 , no. 5 . 
(2002b), «The Migration-Developement Nexus Evidence and Policy Options State-of-the-Art Overview», International Migration, vol. 40, no. 5.

OCHOA-MARín, S. C., S. Cristancho-Marulanda y J. R. Gonzalez-Lopez (2011), «Mujeres compañeras de migrantes: imagen social y búsqueda de servicios de salud sexual y reproductiva», Revista Salud Pública, vol. 13, núm. 2.

OJEDA, N. (2005), «Familias transfronterizas y familias transnacionales: algunas reflexiones», Migraciones Internacionales, vol. 3, núm. 2.

Ordóñez Barba, G. M. (2002), La política social y el combate a la pobreza en México, México, Universidad Nacional Autónoma de México.

PALACIOS ESCOBAR, Á. (2007), «Diferencias, limitaciones y alcances de las estrategias de combate a la pobreza en México», en R. Cordera Campos y C. J. Cabrera Adame (eds.), La politica social en México: tendencias y perspectivas, México, Universidad Nacional Autónoma de México.

Pessar, P. (1999), "Engendering Migration Studies. The Case of New Immigrants in the United States», American Behavioral Scientist, vol. 42, no. 4. (2001), «Women's Political Consciousness and Empowerment in Local, National and Transnatioanal Contexts: Guatemalan Refugees and Returnees», Identities: Global Studies in Culture and Power, vol. 7.

PeSSAR, P. and S. Mahler (2003), «Transnational Migration: Bringing Gender In», IMR, vol. 37 , no. 3 .

PORTES, A. (2009), «Migration and Development: Reconciling Opposite Views», Ethnic and Racial Studies, vol. 32, no. 1.

PORTES, A., P. Fernandez-Kelly and D. Light (2012), «Life on the Edge: Immigrants Comfront the American Health System", Ethnic and Racial Studies, vol. 35, no. 1.

PritCheTT, L. (1997), «Divergence, Big Time», Journal of Economic Perspective, vol. 11, no. 3.

Prospera (2014), «Familias», en https://http://www.prospera.gob.mx/Portal/wb/ Web/padron_de_familias (20 de octubre de 2014).

RAMOS TOVAR, M. E. (2010), «Similitudes y diferencias en la salud psicofísica entre mujeres y hombres migrantes en Estados Unidos. Implicaciones para las políticas de salud y programas de intervención social», en L. Aresti de la Torre (ed.), Mujer y migración, México, Universidad de Nuevo León. 
REICHERT, J. (1981), "The Migrant Syndrome: Seasonal U.S. Wage Labour and Rural Developement in Central Mexico», Human Organization, vol. 40, no. 1. (1982), "A Town Divided: Economic Stratification and Social Relations in a Mexican Migrant Community», Social Problemas, vol. 29, no. 4.

RODRÍGUEZ MEDELLín, L. (2007), «Del asistencialismo a la subsidiaridad: una política de atención a la pobreza», en R. Cordera Campos y C. J. Cabrera Adame (eds.), La política social en México: tendencias y perspectivas, México, Universidad Nacional Autónoma de México.

SANDOVAL, E. (2012), Infraestructuras transfronterizas. Etnografía de itinerarios en el espacio social Monterrey-San Antonio, México, Ciesas y Colef.

SchuTZ, A. (1954), Concept and Theory Formation in the Social Sciences, 257.

Secretaría de Desarrollo Social (2010), Unidad de Microrregiones, http://www. microrregiones. gob. $\mathrm{mx} / \mathrm{zap} / \mathrm{dat}$ enerales.aspx?entra $=$ pdzpyent $=19 \mathrm{ymun}=014$ (20 octubre de 2014).

SEN, A. (1999), Development as Freedom, Nueva York, Anchor Books. (2003a), «La economía política de la focalización», Comercio Exterior, vol. 53, núm. 6. (2003b), «Pobre en términos relativos», Comercio Exterior, vol. 53, núm. 5.

Sørensen, N. N. and K. F. Olwig (2002), Work and Migration: Life and Livelihoods in a Globalizing World, Londres, Nueva York, Routledge.

STARK, O. and R. E. B. Lucas (1988), "Migration, Remmittances and the Family», Economic Development and Cultural Change, vol. 36, no. 3.

SteCklov, G., P. Winter, M. Stampini and B. Davis (2005), «Do Conditional Cash Transfers Influence Migration? A Study Using Experimental Data from the Mexican Progresa Program», Demography, vol. 42, no. 4.

TAYLOR, E. (1999), «The New Economics of Labour Migration and the Role of Remmittances in the Migration Process», International Migration, vol. 37, no. 1.

TOWNSEND, P. (2003), "La conceptualización de la pobreza», Comercio Exterior, vol. 53, núm. 5.

TOYE, J. (2003), «Nacionalizar la agenda contra la pobreza», Comercio Exterior, vol. 53, núm. 6. 
Ullmann, S. H., N. Goldman and D. S. Massey (2011), «Healthier Before they Migrate, Less Healthy when they Return? The Health of Returned Migrants in Mexico», Social Science y Medicine, vol. 73, no. 3.

VALDÉs, G. (1996), Con respeto: Bridging the Distances Between Culturally Diverse Families and Schools: An Ethnographic Portrait, Nueva York, Teachers College Press.

VIZCARRA BORDI, I. y X. Guadarrama Romero (2008), "Cuando los hombres emigran, ¿̇qué ganan y qué pierden las mujeres mazahuas con el programa oportunidades?», en K. Appendini y G. Torres-Mazuera (eds.), ¿̇Ruralidad sin agricultura?, México, El Colegio de México.

Zentgraf, K. M. and N. Stoltz Chinchilla (2012), «Transnational Family Separation: A Framework for Analysis», Journal of Ethnic and Racial Studies, vol. 38, no. 2.

ZÚÑIGA, V. y M. Reyes (1988), «Éxodo rural, estrategias familiares de subsistencia y formas culturales en la Frontera Norte (el caso de Vallecillo, N.L.: el pueblo que se negó a morir)», Bricolage, vol. 1, núm. 100-138.

(2006), «La cultura de los pasaporteados: familia y migración internacional en Vallecillo, Nuevo Leon", El noreste, reflecciones, México, Fondo Editorial Nuevo León.

ZÚÑIGA, V. y J. Sánchez García (2010), «Trayectorias de los alumnos transnacionales en México. Propuesta intercultural de atención educativa", Trayectorias, Revista de Ciencias Sociales, vol. 12, núm. 30. 